\title{
Integrative Structural Biomechanical Concepts of Ankylosing Spondylitis
}

\author{
Alfonse T. Masi, ${ }^{1}$ Kalyani Nair, ${ }^{2}$ Brian J. Andonian, ${ }^{1}$ Kristina M. Prus, ${ }^{1}$ \\ Joseph Kelly, ${ }^{3}$ Jose R. Sanchez, ${ }^{4}$ and Jacqueline Henderson ${ }^{2}$
}

${ }^{1}$ Department of Medicine, University of Illinois College of Medicine, Peoria, IL 61656, USA

${ }^{2}$ Department of Mechanical Engineering, Bradley University, Peoria, IL 61625, USA

${ }^{3}$ Department of Physical Therapy \& Health Science, Bradley University, Peoria, IL 61625, USA

${ }^{4}$ Department of Electrical and Computer Engineering, Bradley University, Peoria, IL 61625, USA

Correspondence should be addressed to Alfonse T. Masi, amasi@uic.edu

Received 31 August 2011; Revised 6 October 2011; Accepted 7 October 2011

Academic Editor: Ruben Burgos-Vargas

Copyright ( $) 2011$ Alfonse T. Masi et al. This is an open access article distributed under the Creative Commons Attribution License, which permits unrestricted use, distribution, and reproduction in any medium, provided the original work is properly cited.

\begin{abstract}
Ankylosing spondylitis (AS) is not fully explained by inflammatory processes. Clinical, epidemiological, genetic, and course of disease features indicate additional host-related risk processes and predispositions. Collectively, the pattern of predisposition to onset in adolescent and young adult ages, male preponderance, and widely varied severity of AS is unique among rheumatic diseases. However, this pattern could reflect biomechanical and structural differences between the sexes, naturally occurring musculoskeletal changes over life cycles, and a population polymorphism. During juvenile development, the body is more flexible and weaker than during adolescent maturation and young adulthood, when strengthening and stiffening considerably increase. During middle and later ages, the musculoskeletal system again weakens. The novel concept of an innate axial myofascial hypertonicity reflects basic mechanobiological principles in human function, tissue reactivity, and pathology. However, these processes have been little studied and require critical testing. The proposed physical mechanisms likely interact with recognized immunobiological pathways. The structural biomechanical processes and tissue reactions might possibly precede initiation of other AS-related pathways. Research in the combined structural mechanobiology and immunobiology processes promises to improve understanding of the initiation and perpetuation of AS than prevailing concepts. The combined processes might better explain characteristic enthesopathic and inflammatory processes in AS.
\end{abstract}

\section{Introduction}

Since 1950, ankylosing spondylitis (AS) had been reported to occur far more prevalently (circa 30 times) among relatives of spondylitis patients than among control subjects [1]. A hereditary risk factor was implicated by such familial findings [1], which have been supported by subsequent studies [2-4]. In 1973, a strong genetic predisposition was confirmed by the discovery of the remarkably high association of AS with HLA-B27 [2, 3].

Beside the recently reviewed expanded genetic risks [4], the multifactorial causation of AS was indicated early by a definite male preponderance of clinical disease [5-8]. The male preponderance was particularly evident among AS patients who had more severe clinical manifestations $[5,8]$. The male-to-female ( $\mathrm{M}: \mathrm{F})$ sex ratio in AS correlates strongly with the severity gradient of disease, which varies from 9:1 in the most progressive patients to a female preponderance in the mildest cases, not explained by presence of the HLA-B27 phenotype [8].

Onset of AS occurs in the first decade in less than 10 percent and after the age of 50 years in only $5 \%$ or less $[6,8]$. The incidence rate rises sharply in puberty $[9]$ or at about the adolescent age of 15 years, reaches a peak onset in the early 20s, and decreases before age $35[8,10]$. Overall, the characteristic age-specific onset pattern of AS is little influenced by the sex effect $[8,10]$. Such risk relations are consistent with the status of myofascial forces or tonicity in 
the body, increasing during adolescent development, peaking in early adulthood, and subsequently decreasing with aging. In the concluding section on Avenues for future research in an early review [6], the suggestion was raised, "also axial muscle tension may be a factor in these degenerative spinal syndromes".

The early suspicion that axial myofascial hypertonicity could predispose to AS [6] has remained consistent with advances achieved in clinical research, immunogenetics, and molecular biology $[10,11]$. However, the hypothesis has not yet been directly confirmed, due to lack of reported quantitative measurements and research on such mechanisms. Accordingly, the aims of this paper are to (1) expand upon our perspectives on the structural biomechanical concept of AS, (2) integrate mechanobiological pathways with current inflammatory concepts, (3) provide a further rationale for encouraging such research, and (4) indicate innovative techniques which may offer promise in future investigations to test the structural biomechanical hypothesis in AS [10].

\section{Enthesis-Related Lesions as Biomechanical Links in the Ankylosing Spondylitis Process}

In AS, the hallmark localization of pathological lesions is at entheses [G. en, in, + thesis, a placing; an insertion] sites, particularly in the vertebral spine [11]. Entheses are the attachments or insertions of tendons, ligaments, or joint capsules into bone $[12,13]$. Enthesitis (G. enthetos, implanted, + -itis, inflammation) was defined [12] as, "traumatic disease occurring at the insertion of muscles where recurring concentration of muscle stress provokes inflammation with a strong tendency toward fibrosis and calcification." Enthesopathy (G. en, in, + thesis, a placing, + pathos, suffering) was defined [12] as "a disease process occurring at the site of insertion of muscle tendons and ligaments into bones or joint capsules". The latter term has gained favor in describing the manifold processes involving entheses, often due to chronic mechanical overloading, with or without evidence of inflammation [13].

The target enthesis lesions in AS are generally interpreted to result from inflammatory mechanisms associated with biomechanical stress at the local level [14]. The axial myofascial hypothesis proposes a centralized mechanism for increased tensional stresses in the postural musculoskeletal anatomy that can transmit excessive forces to entheses in the spine and girdles $[15,16]$. A normally relaxed muscle is relatively soft and extensible. It can efficiently dissipate stress concentrations by transferring or absorbing them [13]. To the contrary, stiffer muscles less effectively absorb or distribute forces [13]. Thus, stiffer and tenser muscles could likely transmit greater stresses to tendons or ligaments and to bony enthesis sites, which serve an anchoring role $[13,15]$.

This perspective concentrates on enthesis lesions in AS, since they have received greater attention in recent research $[10,11]$ than a preceding review of sacroiliac joint (SIJ) involvement [16]. The mechanical principle of integrated tension (tensegrity) [17-19] is fundamental to the structural hypothesis. Application of the tensegrity theory to axial myofascial hypertonicity in the postural system can theoretically rationalize concurrently excessive tensional forces on entheses coexisting with increased compressional loads across the SIJs $[16,19]$. Anatomical cross-bracing of the dorsal and lateral postural myofascial support systems could overload the SIJs by increased compressional forces and stiffness (Figure 1) [16]. Hypertonicity of the deeper vertebral myofascial system, like the lumbar multifidus muscles, could exert its main effects on increased tensional forces at the spinal entheses and manifest as symptomatic and objective postural stiffness [15].

\section{Human Resting Muscle/Myofascial Tone (HRMT)}

Axial or postural human resting muscle/myofascial tone (HRMT) is an innate polymorphic trait which is independent of the central nervous system control and contributes vitally to stability in balanced equilibrium positions $[15,20$, 21]. Increased low-back muscle stiffness and tightness in patients with early AS was first reported by Forestier et al. in 1951 [22], which was called the "bowstring sign" [22]. That observation was subsequently confirmed by palpation and electromyography studies, as reviewed [23].

The concept of HRMT is believed to incorporate an innate individual variability (polymorphism), particularly of the axial postural system $[15,24]$. Variability is also likely to be clinically relevant to proximal limb muscles and girdles, but less so for the distal extremities. This paper incorporates HRMT into the biomechanical hypothesis only as related to the axial postural system effects on vertebral entheses and the SIJs $[10,16]$.

\section{An Early Historical Interpretation of Clinical Syndromes at Enthesis Sites}

Mechanisms causing lesions at enthesis sites are complex [13, 14]. A paucity of documentation exists on the evolution of the processes, from initiation, through their course, and into the later healing stages. The first PubMed reference found on enthesitis was from 1959, titled as "Enthesitis-traumatic disease of insertions" [25]. The tissue reactions at insertions were described as follows: "most frequently these stimuli are of traumatic, particularly microtraumatic, origin. The continually recurring concentration of muscle stress at these points provokes a reaction of inflammation with a strong tendency to the formation of fibrosis and calcification" [25].

\section{Evolution of Histological Features in Enthesis Lesions: Stages of Erosion, Then Repair}

The earliest pathoanatomical review of enthesopathy found in PubMed was from 1966 [26], which included description of both peripheral ligamentous and vertebral lesions. The enthesis was referred to as a unit [26] and is now referenced as an organ [13,27]. The enthesis unit was defined as a special 


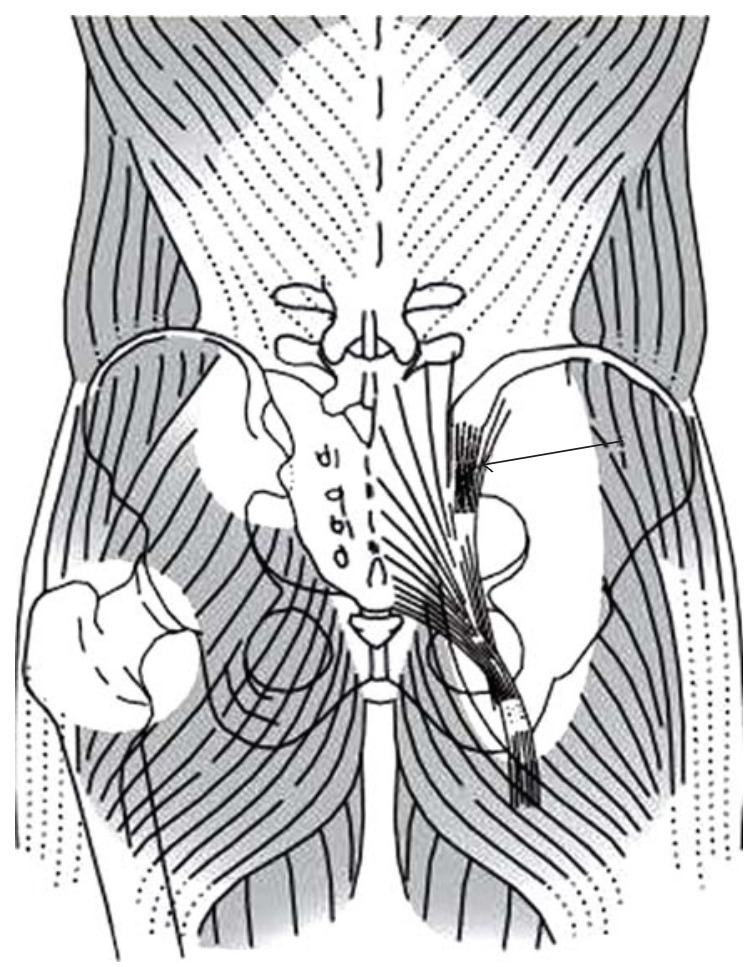

Figure 1: Posterior anatomical relations of the lumbopelvic region. On the left side, the upper window shows the sacroiliac joint (SIJ). The lower window shows how the hip joint is interposed between posterior cross-braced muscles and how the pelvis is stabilized. The right side shows the attachment of the hamstrings (particularly the biceps tendon) to the sacrotuberous ligament. The arrow indicates the long dorsal sacroiliac ligament (LDSIL). Axial myofascial hypertonicity could theoretically exert increased compressional forces on the pelvis, SIJs, and hips. (The original was published as Figure 14.4 in [16], In: A. Vleeming, V. Mooney, R. Stoeckart, eds. Movement, Stability, and Lumbopelvic Pain: Integration of Research and Therapy. Edinburgh, Scotland: Churchill Livingston; 2007:205-227, reproduced with permission from Churchill Livingston, Copyright Elsevier, 2011).

anatomical formation consisting of (1) a specifically structured part of the bone, (2) the transitional part of the tendon, and (3) interpolated fibrocartilage, which is partially calcified [26]. This definition has essentially held true [13, 27]. An absence of periosteum at the insertion sites of tendon was emphasized as well as an exceptionally great strain at the terminal part of the tendon [26]. A metaplastic process was described at the insertional zone that was considered to be a possible adaptive manifestation to "one-sided overstrain." Often, the alterations included concurrent inflammatory changes and new bone formation, as in a reparative hyperplastic stage [26].

This group [26] described localized granular inflammatory changes in loose, richly vascularized areolar tissue of the ligamentous enthesis. At this stage, the localized bone cortex had become thinned and manifested marginal defects [26]. These findings were considered to be the destructive (erosive) phase of enthesitis. Notably, reparative changes were observed along with the destructive ones. In the advanced stage, typical ossifying enthesitis (enthesophyte) had developed [26].

\section{Enthesis Lesions in Ankylosing Spondylitis}

The classical reported pathoanatomical findings in AS patients are almost uniformly restricted to advanced stages in the available tissue materials, usually in late and post-mortem stages [28-32]. Initial lesions of the local processes are hardly documented [29].

The classical paper on enthesopathy in AS was the subject of the Heberden Oration, 1970 [31]. Firstly, Ball [31] compared rheumatoid spondylitis (RS), mainly defined from early cervical involved cases, to the histopathology of late AS spondylitis. In the cervical RS cases, instability and dislocation were stated to be associated with erosive synovitis of the apophyseal joints and to be associated with destructive lesions of the corresponding disc [31]. The enthesopathy of RS occurred in the annulus fibrosus of cervical discs and was considered to be secondary to the primary synovitis. It was not associated with prominent reactive bone formation, as was found in AS [31]. Immobilization was considered as a mechanism in the prominent enchondral ossification feature in AS, which occurs in the apophyseal joints [31].

Secondly, Ball [31] examined extraspinal ligamentous lesions of AS in mainly biopsy specimens. Like Niepel et al. [26], the striking findings were multiple focal microscopic inflammatory lesions localized to the ligamentous attachments [31]. The whole or greater part of the enthesis was destroyed in association with small erosions or defects in the cortical bone. The inflammation reaction was considered brief in individual lesions, mostly involving lymphocytes and plasma cells [31]. The erosive lesions healed by deposition of reactive (woven) bone in a finely fibrous connective tissue without preceding cartilage formation. The new bone tended to fill in the cortical defect as well as having joined the deeper bone to the eroded end of the ligament. The new bone formed a new enthesis above the original level of the cortical surface. In the final healing stage, it appeared as a small irregular bony prominence [31].

\section{Intervertebral Disc Lesions in Ankylosing Spondylitis}

According to Ball [31], the concept that ossification of the intervertebral discs in AS is inflammatory in origin may be traced back to Engfeldt et al. [28] and to Bywaters [29, 30]. Currently, inflammation is still considered to be essential to the pathogenesis of AS [10, 11]. Ball [31] interpreted the spinal lesions from 13 necropsy specimens, 12 having deceased after 12 to 33 years following onset of AS. The late and somewhat fragmentary nature of the spinal specimens was acknowledged [31].

Ball [31] found erosive lesions at the anterior or anterolateral attachments of the outer fibers of the annulus at the corner of the vertebral body and at the junction of the annular flange. Infiltration of lymphocytes and plasma cells was 
scanty in some and prominent in other lesions. Of note, the common longitudinal ligament was conspicuously unaffected in anterior lesions. In the area of the erosive lesions, the vertebral cortex was replaced by a narrow layer of reactive bone that spread for a short distance into the outer annulus, that is, the disc syndesmophyte. The process involved the replacement and remodeling of the reactive bone into mature (lamellar) bone. Ball [31] tentatively interpreted a mechanism for the growth of disc syndesmophytes as "the intermittent occurrence of inflammatory lesions in previously stable syndesmophytes." Our tentative biomechanical interpretation of this process might be that an inflammatory phase, possibly contributed by microinjury, precedes the ossification (healing) or syndesmophyte (immobilization) stage.

Lastly, Ball [31] considered the ossification process in the apophyseal joints. In two AS cases, capsular ossification occurred in the setting of doubtful erosive synovitis and with preserved articular cartilages. The capsular findings were interpreted as equivalent to syndesmophyte formation and that the enclosed articular cartilages were being replaced by a nonspecific process of enchondral ossification [31].

In subsequent reviews of the articular pathology of ankylosing spondylitis, Ball [32-34] raised the possibility that inflammatory enthesopathy was not the only mechanism that may give rise to syndesmophyte formation. This process can be attributed to implied immobilization and changes in load transmission across the disc [33]. Destructive spondylodiscitis lesions were also reviewed in the later articles [32, 33], which had not been earlier considered [31]. Clinical, radiological, and pathological evidence indicated that these destructive spondylodiscitis lesions are essentially owing to trauma in a spine that for various reasons is susceptible to stress [32, 33]. Controversy exists, however, regarding an interpretation of the variable histopathology of the spondylodiscitis lesions, which may not show inflammatory infiltration $[32,33]$.

\section{A Novel Biomechanical Interpretation of Classical Histological Studies of Enthesis Lesions}

Injury mechanisms from structural impacts were only later considered by Ball $[32,33]$. Microinjury was not addressed in relation to causing erosive lesions at the attachment sites of the annulus fibrosus to the vertebral margins, at which syndesmophytes start to grow [31, 32]. Modern biomechanical research has established that this disc-vertebral interface is subjected to increased localized force concentrations at its outer circumferential boundaries in the spine [35].

Further research into the indicated differential involvement of the lumbar anterior longitudinal ligament (ALL) versus the disc entheses in AS $[28,31]$ could potentially clarify mechanistic pathways in syndesmophyte formation. Man$y$ reports stated that the anterior ossification between the vertebral bodies is not situated in the ALL, but rather in the periphery of the disks $[28,31]$. Both tissues are in close proximity to the cellular infiltrations and new bone formation at the edge of the annulus [31, plate 3(a)]. A hypothetical question might be raised as follows. Could differential degrees of mechanically induced microinjury, in one versus the other tissue, lead to the observed differences in inflammatory reactions? If so, might such mechanisms influence later bony proliferation leading to differential involvements in syndesmophyte formation? Such biomechanical issues had not been raised in the classical papers [28-33] nor have we encountered them in the current literature.

\section{Parallels in Tendinopathy and Ankylosing Spondylitis Enthesis Lesions}

Tendinopathy is currently considered to be mainly noninflammatory (enthesopathy) [13, 36, 37], whereas AS is thought to have a primarily inflammatory cause (enthesitis) [11]. Mechanical overloading is believed to be the primary initiating pathology in degenerative and overuse tendinopathy $[38,39]$. Like AS [40], many tendinopathies are insertional in nature, having a disease localization at enthesis sites, where the tendon or ligament attaches to bone [37]. One may question if the pathways leading to these conditions might be more similar than previously believed. Might biomechanical stress mechanisms also be an important predisposing pathway in AS?

Histopathological studies of the Achilles tendinopathy are typically characterized by a lack of inflammatory cells and other evidence for inflammatory mechanisms [13, 41, 42]. Rather, they show a poor healing response, involving collagen fiber disorientation, tenocyte hypercellularity, neovascularization, and neurovascular ingrowth [38, 42]. Calcification and osseous metaplasia can also be found in symptomatic tendons [38]. A primarily noninflammatory mechanism is also endorsed by the limited efficacy of anti-inflammatory drug therapy of tendinopathy [43].

Bone formation can occur at enthesis (enthesophyte) sites in tendinopathy disorders and differs from normal fracture healing [41]. Enthesophytes have been shown to form in regions of high tensile forces, thereby increasing contact surface area between the tendon or ligament and bone [44, 45]. Anatomical connections from entheses to neighboring synovium and bone may explain how chronic biomechanical stress at these sites may lead to secondary histopathology in AS, including synovitis and bone marrow edema [44-46].

\section{Clinical and Cytokine Parallels in Tendinopathy and Ankylosing Spondylitis}

Mechanical forces interacting with cytokines, such as TGF- $\beta$ and IL- $1 \beta$, can stimulate extracellular matrix (ECM) gene and protein expression, leading to either anabolic or catabolic pathways [47]. Excessive mechanical forces may result in tissue microinjury in tendinopathy and in AS. Such stressmediated processes could activate inflammatory responses by release of damage-associated molecules, cytokines, or other mediators $[44,48]$. In overuse tendinopathy, an early increase in proinflammatory cytokine levels is believed to contribute to the observed later-stage degenerative changes 
[49]. Few human data are available on cytokine influences upon enthesis lesions in AS.

Athletes with symptomatic tendinopathy have been shown to have stiffer Achilles tendons than normal control subjects [50]. Individuals with diabetes mellitus are also predisposed to the Achilles tendinopathy, possibly related to structural abnormalities associated with increased stiffness [51]. Perhaps, greater myofascial stiffness could generally predispose individuals to enthesopathy, including tendinopathy and AS $[13,15,37]$ ?

\section{Comparison of Demographic Profiles and Implications of Treatment Responses}

Similarities in host profiles of TP and AS patients could lend support to biomechanical pathways in both disorders. Overall, AS and patellar tendinopathy occur about twice as often in men as in women $[8,38,39,52]$. Men have greater musculotendinous stiffness than women, which correlated positively with muscle mass [53]. Men have greater predisposition to bony tensional attachment injuries, whereas women are more susceptible to joint instability and intra-articular injury, such as anterior cruciate ligament (ACL) rupture $[53,54]$.

Although inflammation is not considered to be the primary cause of tendinopathy, local morning stiffness which eases with activity is commonly observed [38]. Such symptoms are analogous to the pattern of chronic inflammatorytype back pain in AS [55]. Spinal mobility is restricted in early AS [56] and reduced muscular flexibility is also thought to contribute to the development of patellar tendinopathy in athletic populations [57].

Recommendations for management of tendinopathy [13, 43] and AS [58-60] both include physical therapy interventions to maximize the long-term quality of life, although anti-inflammation therapy has an added, relatively greater benefit in AS than in tendinopathy $[11,58]$. The combination of regular, active exercise and pharmacological treatment has been shown to be more effective in the long-term than usual therapy in AS [59]. Thus, physical rehabilitation approaches are beneficial in both conditions. The difference in benefits from anti-inflammatory therapy between TP and AS may reflect relative degrees of biomechanical versus immunological pathways in the respective conditions $[10,11]$.

If increased axial myofascial tonicity is confirmed in AS $[10,22,23]$, then the transmitted enthesis tissue stiffness could result in greater microinjury. Such pathway deserves further investigation and is consistent with beneficial results of structured rehabilitation interventions in AS, such as global postural reeducation [61] and active exercise treatments [59].

\section{Mechanobiology and Immunobiology Interactions in Ankylosing Spondylitis: Figure 2}

Trauma was proposed as a possible initiating stimulus for chronic interactions of the innate and adaptive arms of the immune system in spondyloarthritis (SpA) and AS [14, 48, 62 . Tissue microinjury could locally activate the innate immune system by release of damage-associated molecules, cytokines, or other mediators [44, 45, 62]. In normal subjects, proinflammatory cytokine release is increased in skeletal muscle following eccentric exercise [63]. However, in the same normal subjects, a circulating systemic proinflammatory response is not seen, possibly due to activation of antiinflammatory cytokines [63].

We agree with others $[14,48,64]$ that microtrauma and biomechanical stress may be important initiating triggers and chronic perpetuating stimuli in AS. Our hypothesis of an innate axial (spinal) myofascial hypertonicity $[10,15,16]$ provides a theoretical framework for increased axial stiffness in AS. In turn, it could predispose to exaggerated stress transmissions through entheses, leading to greater micro-damage and abnormal tissue repair responses [15, 16, 47]. A constitutional biomechanical diathesis, in combination with a proinflammatory predisposition and other genetic factors, could initiate and perpetuate inflammation as well as osteoproliferation (syndesmophyte) formation in the AS patient (Figure 2) [16, 64, 65].

Mechanotransduction translates intrinsic and extrinsic forces into cellular and molecular responses $[18,47,66]$. Accordingly, cells modify their responses to varied stress by mechanobiology pathways [66]. Increased structural and entheseal stresses on extracellular matrix (ECM), tendon, and bone $[16,66]$ of AS patients could be expected to result in altered and pathological tissue responses $[15,16,37,44$, 66]. In an in vivo rodent tendon model, mechanical stress can alter the expression of IL- $1 \beta$ in a load-dependent fashion [67]. In bone, osteoblasts are also load-sensitive cells and can produce bone morphogenic proteins (BMPs) [66], which were found to be increased in a murine model of ankylosing enthesitis [64, 68]. Moreover, microtrauma can release cartilaginous molecules that activate inflammation by pattern recognition receptors $[48,69]$. Those released ECM fragments can be incorporated intracellularly and initiate a proinflammatory cascade [69].

\section{Complex Interactions of Wnt, DKK-1, BMPs, and TNF in Murine Models}

Investigation into TNF mechanisms in the complex bony proliferation process has revealed relations to other molecular pathways. The Wnt family members are relevant, as some induce differentiation of osteoblasts and block osteoclast activity, resulting in a net gain of bone [70]. Dickkopf-1 $(\mathrm{DKK}-1)$ is an antagonist of the $\mathrm{Wnt} / \beta$-catenin bone-forming pathway. Excess TNF, as commonly occurring in AS patients, has been found to stimulate the production of DKK-1 [71]. In a murine inflammatory arthritis model, DKK-1 inhibition reversed bone erosion into osteophyte formation, suggesting that DKK-1 is an important inhibitory molecule in bone formation [71]. Another study found increased serum DKK-1 levels in AS patients treated with anti-TNF agents as compared to rheumatoid arthritis patients and normals [72]. These findings indicate a paradox in the osteoproliferation mechanisms in AS. 


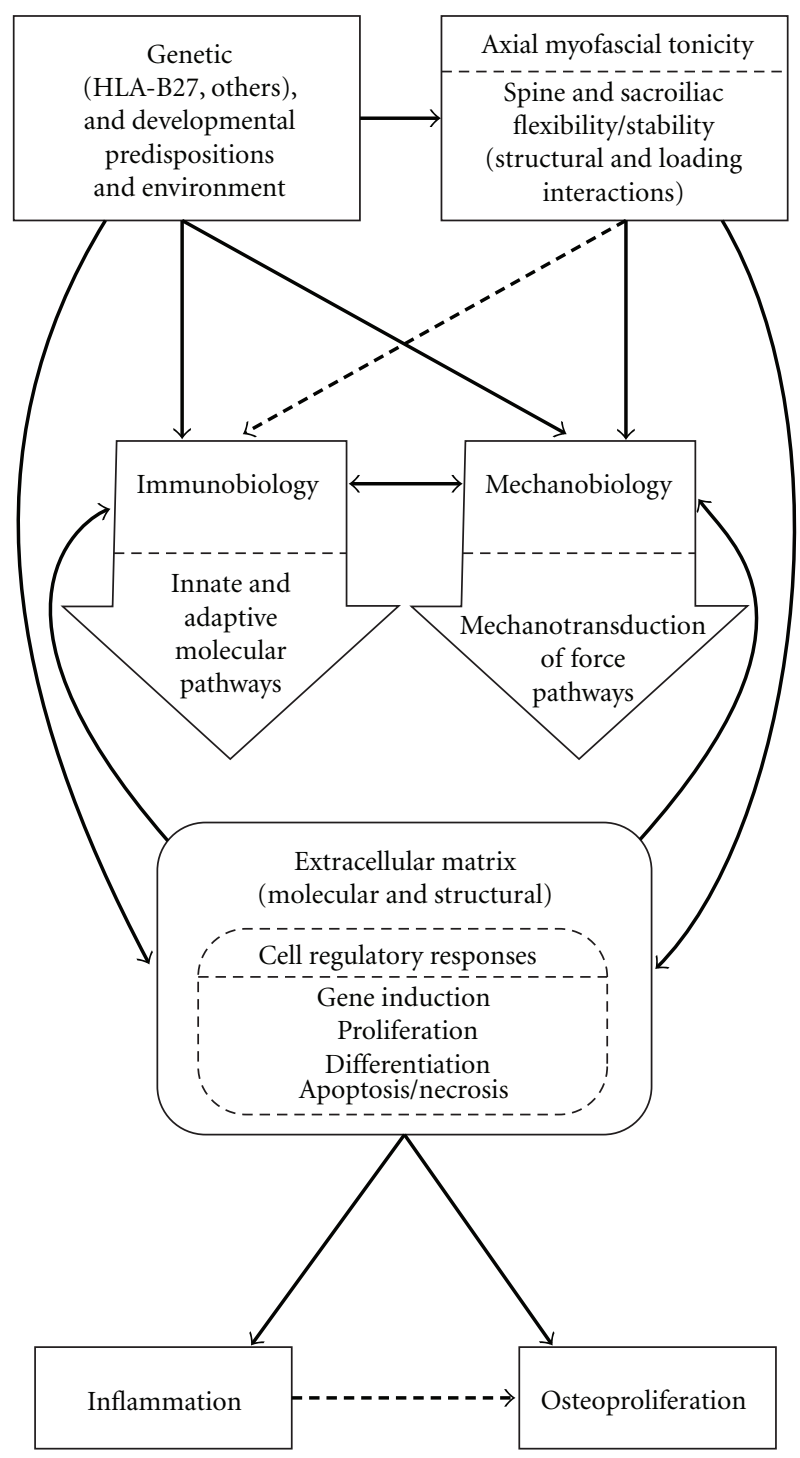

FIgURE 2: A theoretical outline of immunobiology and mechanobiology processes interacting in initiation and perpetuation of pathways in ankylosing spondylitis. (Reproduced with permission for reprinting from the Journal of Rheumatology; the original was published as a Figure in [10], A. T. Masi, 2011; 38 (10): (pp. $2092-$ 2094)).

The TNF-brake hypothesis [73] may offer a possible explanation of the bony proliferative process in AS. It proposes that, after TNF blocking agents are administered, the DKK-1 is less stimulated by TNF, and then Wnt family members are less inhibited, permitting increased bony proliferation [73]. It has been further proposed that, with increase of Wnt member signaling, DKK-1 may be increased as a feedback balancing response [72].

Bone morphogenetic proteins (BMPs) also have a role in osteoproliferation. The BMPs control bone growth and are inhibited by noggin gene transfer [68]. TNF can also stimulate certain BMPs [68]. Accordingly, overexpression of BMPs may stimulate greater bone formation at damage sites and may contribute to osteophyte formation [74]. Since AS patients may have increased TNF levels, BMP could be upregulated and may contribute to bony proliferation. One study found a nonsignificant increase in serum BMP-7 levels in AS patients [75], and increased serum levels of BMP-2 and BMP-7 were found in another study of AS patients [76].

\section{Mechanical Stress Activation of Inflammatory Pathways}

Mechanical stress can become translated to inflammation and cellular responses via molecular induction pathways $[18,47,66]$. Cyclooxygenase-2 (Cox-2) can be induced by mechanical stress, which leads to increased prostaglandin E2 (PGE2) synthesis in cartilage, as commonly seen in OA [77]. Also, the intracellular NALP3 pathway can translate mechanical or metabolic stimuli into cellular responses [69]. In this pathway, mechanical or metabolic stress can release molecules related to cellular damage. These cellular stress molecules can be sensed by membrane receptors or be endocytosed, leading to NALP3 activation. This molecule is part of the nucleotide-binding domain, leucine-rich repeat containing family [69]. It is related to increases in proinflammatory cytokines, such as TNF [69]. This pathway culminates in increased levels of the proinflammatory cytokine, interleu$\operatorname{kin}-1 \beta[69]$.

These Cox-2 and NALP3 transduction pathways may possibly explain how increased mechanical loading may lead to inflammation and bony proliferation. Both pathways induce increased proinflammatory cytokine release [69, 77] and are consistent with increased BMP upregulation. In a review, Lories et al. [64] proposed that TNF stimulates BMP and DKK-1. While DKK-1 inhibits osteoblast differentiation by suppressing Wnt family members, the BMP augmentation may be an even greater stimulus for bone formation [64]. One study indicated that anti-TNF treatment was more clinically effective in patients with shorter disease duration [78]. It was suggested that disease modification is required at the earliest stages possible to avoid increased levels of BMP or sensitization to Wnt members and greater osteoproliferation [78].

Recent research has indicated that tissue inflammatory reactions, as is found in rheumatoid arthritis (RA) and in AS lesions, can result from nonspecific cytokine stimulation, including T-helper cell activation [79]. Accordingly, such tissue reactions need not to be autoimmune nor dependent upon specific antigen activations. Rather, such inflammatory reactions in these diseases may be initiated and contributed by cytokines, possibly released from micro injury contributed by an innate biomechanical diathesis. In such scenario, excessive axial HRMT could be a structural body variant that may predispose to enhanced entheseal microinjury and initiation of inflammatory pathways $[10,15]$. Of potential interest, a recent report indicated marked (up to 100-fold) upregulation of gene transcripts related to myocyte/myofibroblast biology in synovial tissue samples from SpA versus RA arthritis patients, which suggested structural remodeling mechanisms in SpA [80]. 


\section{Significance of Research on Axial HRMT and Critical Barriers to Progress}

Myofascial tone is literally the tension or stiffness of these specialized tissues which is universally recognized to accompany movements and resistive activities. In the axial (spinal) system, myofascial tone is the primary contributor to stability in various postural activities, far greater than the osteoligamentous component $[15,20]$. Myofascial tone exists in the body, whether it is (1) passive or active (2) in postural balance or unbalanced, and (3) static (resting) or in motion. Accordingly, the body may be considered as being in a prestressed architectural design, which is labeled as biotensegrity $[17,18]$. Notably, myofascial tone may encompass intrinsic passive properties of the tissues as well as an active contractile component which is superimposed under the control of the central nervous system (CNS) [15, 19-21]. The passive, resting (static) tone is independent of CNS control. It results from the elastic mechanical properties of the stable crossbridges between the actin and myosin filaments of muscle fibers and the integrated connective tissue filaments $[15,20$, 21].

A critical problem in the interpretation of clinical studies of HRMT has been the differentiation of the static, passive condition alone from any additional low-level contraction superimposed by CNS activation [81]. Such confounding of the passive properties by the low-level active contraction component has led to confusion and misinterpretation in studies of the human physiology and clinical relevance of myofascial tone $[15,20,21]$. We endorse research to accurately characterize the low-level, passive biomechanical properties of axial HRMT (EMG-silent) in relation to AS risks. The intrinsic HRMT viscoelastic property is a vital body trait which has been overlooked [82] or misinterpreted [15, 20, 21]. Current lack of confirmed quantitative data on individual variability (polymorphism) of axial HRMT now warrants critical study of its physical properties and relevance to body biomechanics in AS.

\section{Quantifying Axial HRMT (EMG-Silent)}

Emerging methodological techniques can noninvasively quantify the viscoelastic (elasticity, stiffness, and tension) properties of myofascia at precise body levels. Such measurements have not yet been reported at the lumbar level. Concurrent surface electromyography (sEMG) should be performed at the measurement loci to confirm the passive status and absence of superimposed CNS activation. Extensive data exist on spinal muscle properties in various degrees of activated contraction, but none for comparison at rest [82].

The lumbar spine is stabilized and moved by sets of muscles that have varying biomechanical roles [15, 83]. The core muscles provide mainly segmental stability, whereas more peripheral muscles control mainly global movements and active stabilization of the trunk $[15,83,84]$. Based upon such biomechanical principles, our hypothesis is that the core muscles, for example, lumbar multifidus, will contribute mainly to variability in innate spinal myofascial stiffness among individuals (polymorphism). Some polymorphic variation is also expected in the more superficial muscle networks, for example, erector spinae.

Techniques have existed that can quantify muscle tone $[81,85]$. However, they are basically dynamic, operating at macroanatomical levels via imposed passive joint movements (calf, thigh, or the trunk). Emerging technology, like the Myoton instrument $[86,87]$ and ultrasonic shear wave elastography [50,88-90] will enable noninvasive quantification of resting muscle properties of elasticity, stiffness, and tension at precise lumbar anatomical levels. The Myoton range of depth measurements is mainly limited to several centimeters within the more superficial muscles, for example, erector spinae. Ultrasonic shear wave elastography technique will be required to measure the deeper or core lumbar muscles, for example, the multifidus [90]. The use of separate measurement techniques on the superficial (Myoton and elastography) and deeper (elastography) lumbar muscles will aid in interpreting the respective findings.

Cross-sectional analysis of axial HRMT could test whether or not AS patients have greater lumbar myofascial biomechanical properties of stiffness/tension than normal subjects or even greater than patients with non-AS chronic, mechanical low-back disorders [15]. The ultrasonic shear wave elastography technique is able to scan deeply into the dorsolumbar myofascial tissues to investigate viscoelastic properties of the multifidus at the L3-L4 level and of its enthesis sites. These viscoelastic properties have not been reported and may become valuable as a biomarker in an earlier diagnosis of AS. Cost-effective biomechanical criteria for AS have not yet been investigated, which may help to identify its early stage $[22,23]$. Viscoelastic properties may have utility in also helping to identify asymptomatic high-risk susceptibles who may be likely to develop AS, such as those HLA-B27-positive firstdegree relatives of AS probands [15].

\section{Technical Innovation Is Needed to Quantify Biomechanical Influences on AS}

Interdisciplinary biomechanical approaches are needed to quantify and analyze passive (EMG-silent) axial (spinal) myofascial tone in normal subjects, patients with AS, and those with other chronic LBP conditions. Quantitative biomechanical study is needed to analyze individual variability characteristics (polymorphisms) of the hardly appreciated axial HRMT trait in large samples of healthy persons. Such studies will reveal and elucidate the significance of resting axial muscle tone in the spinal musculoskeletal system.

When axial (and general body) passive tone and stiffness are normally sufficient, this trait is biomechanically and metabolically efficient in energy expenditure [19]. Insufficient or excessive axial HRMTs are likely to have physical penalties, like increased risks of developing spinal consequences [24] and possible energy metabolic impacts [19]. Axial HRMT is a macrostructural component of the body tensegrity design [17-19]. Incorporation of tensegrity [17-19] concepts and utilization of structural mechanical modeling [35] promises to enhance understanding of spinal disorders, like AS. 


\section{Conflict of Interests}

The authors declare that they have no conflict of interests.

\section{Acknowledgments}

The authors thank Becky Hawkins for her invaluable technical assistance in completing this paper. Support for this project was provided by the Department of Medicine, University of Illinois College of Medicine at Peoria, and by the MTM Foundation.

\section{References}

[1] A. H. Hersh, R. M. Stecher, W. M. Solomon, R. Wolpaw, and H. Hauser, "Heredity in ankylosing spondylitis; a study of fifty families," American Journal of Human Genetics, vol. 2, no. 4, pp. 391-408, 1950.

[2] D. A. Brewerton, F. D. Hart, A. Nicholls, M. Caffrey, D. C. James, and R. D. Sturrock, "Ankylosing spondylitis and HL-A 27," The Lancet, vol. 1, no. 7809, pp. 904-907, 1973.

[3] L. Schlosstein, P. I. Terasaki, R. Bluestone, and C. M. Pearson, "High association of an HL-A antigen, W27, with ankylosing spondylitis," The New England Journal of Medicine, vol. 288, no. 14, pp. 704-706, 1973.

[4] M. A. Brown, "Genetics of ankylosing spondylitis," Current Opinion in Rheumatology, vol. 22, no. 2, pp. 126-132, 2010.

[5] A. T. Masi, "HLA B27 and other host interactions in spondyloarthropathy syndromes," Journal of Rheumatology, vol. 5, no. 4, pp. 359-362, 1978.

[6] A. T. Masi, "Epidemiology of B27-associated diseases," Annals of the Rheumatic Diseases, vol. 38, supplement 1, pp. suppl 131-134, 1979.

[7] A. T. Masi and T. A. Medsger, "A new look at the epidemiology of ankylosing spondylitis and related syndromes," Clinical Orthopaedics and Related Research, vol. 143, pp. 15-29, 1979.

[8] A. T. Masi, "Do sex hormones play a role in ankylosing spondylitis?" Rheumatic Disease Clinics of North America, vol. 18, no. 1, pp. 153-176, 1992.

[9] M. Wilkinson and E. G. Bywaters, "Clinical features and course of ankylosing spondylitis; as seen in a follow-up of 222 hospital referred cases," Annals of the Rheumatic Diseases, vol. 17, no. 2, pp. 209-228, 1958.

[10] A. T. Masi, "An added perspective on the 2009 SPARTAN and IGAS report: an innate axial myofascial hypertonicity," Journal of Rheumatology, vol. 38, no. 10, pp. 2092-2094, 2011.

[11] J. D. Reveille and D. O. Clegg, "Prologue: 2009 Joint meeting of Spondyloarthritis Research and Therapy Network (SPARTAN) and International Genetics of Ankylosing Spondylitis (IGAS)," Journal of Rheumatology, vol. 37, no. 12, pp. 2604-2605, 2010.

[12] Stedman's Medical Dictionary, Williams \& Wilkins, Baltimore, Md, USA, 26th edition, 1995.

[13] M. Benjamin, H. Toumi, J. R. Ralphs, G. Bydder, T. M. Best, and S. Milz, "Where tendons and ligaments meet bone: attachment sites ('entheses') in relation to exercise and/or mechanical load," Journal of Anatomy, vol. 208, no. 4, pp. 471-490, 2006.

[14] D. McGonagle, L. Stockwin, J. Isaacs, and P. Emery, "An enthesitis based model for the pathogenesis of spondyloarthropathy. Additive effects of microbial adjuvant and biomechanical factors at disease sites," Journal of Rheumatology, vol. 28, no. 10, pp. 2155-2159, 2001.
[15] A. T. Masi, K. Nair, T. Evans, and Y. Ghandour, "Clinical, biomechanical, and physiological translational interpretations of human resting myofascial tone or tension," International Journal of Therapeutic Massage \& Bodywork, vol. 3, pp. 16-28, 2010.

[16] A. T. Masi, M. Benjamin, and A. Vleeming, "Anatomical, biomechanical, and clinical perspectives on sacroiliac joints: an integrative synthesis of biodynamic mechanisms related to ankylosing spondylitis," in Movement, Stability, and Lumbopelvic Pain: Integration of Research and Therapy, A. Vleeming, V. Mooney, and R. Stoeckart, Eds., pp. 205-227, Churchill Livingstone, Edinburgh, Scotland, 2007.

[17] D. E. Ingber, "The architecture of life," Scientific American, vol. 278, no. 1, pp. 48-57, 1998.

[18] D. E. Ingber, "Mechanobiology and diseases of mechanotransduction," Annals of Medicine, vol. 35, no. 8, pp. 564-577, 2003.

[19] A. T. Masi and E. G. Walsh, "Ankylosing spondylitis integrated clinical and physiological perspectives," Clinical and Experimental Rheumatology, vol. 21, no. 1, pp. 1-8, 2003.

[20] A. T. Masi and J. C. Hannon, "Human resting muscle tone (HRMT): narrative introduction and modern concepts," Journal of Bodywork and Movement Therapies, vol. 12, no. 4, pp. 320-332, 2008.

[21] S. Mense and A. T. Masi, "Increased muscle tone as a cause of muscle pain," in Muscle Pain: Understanding the Mechanisms, S. Mense and R. D. Gerwin, Eds., pp. 207-249, Springer, Berlin, Germany, 2010.

[22] J. Forestier, F. Jacqueline, and J. Rotesquerol, La Spondylarthrite Ankylosante: Clinique, Radiologie Anatomie Pathologique, Traitement, Masson \& Cie, Paris, France, 1951.

[23] A. T. Masi, S. Sierakowski, and J. M. Kim, "Jacques Forestier's vanished bowstring sign in ankylosing spondylitis: a call to test its validity and possible relation to spinal myofascial hypertonicity," Clinical and Experimental Rheumatology, vol. 23, no. 6, pp. 760-766, 2005.

[24] A. T. Masi, J. L. Dorsch, and J. Cholewicki, "Are adolescent idiopathic scoliosis and ankylosing spondylitis counteropposing conditions? A hypothesis on biomechanical contributions predisposing to these spinal disorders," Clinical and Experimental Rheumatology, vol. 21, no. 5, pp. 573-580, 2003.

[25] G. La Cava, "Enthesitis-traumatic disease of insertions," JAMA, vol. 169, pp. 254-255, 1959.

[26] G. A. Niepel, D. Kostka, S. Kopecky, and S. Manca, "Enthesopathy," Acta Rheumatol Balneol Pistiniana, vol. 1, pp. 5-64, 1966.

[27] M. Benjamin and D. McGonagle, "The anatomical basis for disease localisation in seronegative spondyloarthropathy at entheses and related sites," Journal of Anatomy, vol. 199, no. 5, pp. 503-526, 2001.

[28] B. Engfeldt, R. Romanus, and S. Yden, "Histological studies of pelvo-spondylitis ossificans (ankylosing spondylitis) correlated with clinical and radiological findings," Annals of the Rheumatic Diseases, vol. 13, no. 3, pp. 219-228, 1954.

[29] E. G. L. Bywaters, VIth European Congress of Rheumatology, Instituto Portugues de Reumatologia, Lisbon, Portugal, 1967.

[30] E. G. Bywaters, "The early lesions of ankylosing spondylitis," Annals of the Rheumatic Diseases, vol. 28, no. 3, p. 330, 1969.

[31] J. Ball, "Enthesopathy of rheumatoid and ankylosing spondylitis," Annals of the Rheumatic Diseases, vol. 30, no. 3, pp. 213223, 1971.

[32] J. Ball, "Articular pathology of ankylosing spondylitis," Clinical Orthopaedics and Related Research, vol. 143, pp. 30-37, 1979. 
[33] J. Ball, "Pathology and pathogenesis," in Ankylosing Spondylitis, J. M. H. Moll, Ed., chapter 9, pp. 9-12, Churchill Livingstone, Edinburgh, Scotland, 1980.

[34] J. Ball, "The enthesopathy of ankylosing spondylitis," British Journal of Rheumatology, vol. 22, no. 4, supplement 2, pp. 2528, 1983.

[35] M. A. Adams, P. Dolan, and D. S. McNally, "The internal mechanical functioning of intervertebral discs and articular cartilage, and its relevance to matrix biology," Matrix Biology, vol. 28, no. 7, pp. 384-389, 2009.

[36] K. M. Khan, J. L. Cook, P. Kannus, N. Maffulli, and S. F. Bonar, "Time to abandon the "tendinitis" myth," British Medical Journal, vol. 324, no. 7338, pp. 626-627, 2002.

[37] H. M. Shaw and M. Benjamin, "Structure-function relationships of entheses in relation to mechanical load and exercise: review," Scandinavian Journal of Medicine and Science in Sports, vol. 17, no. 4, pp. 303-315, 2007.

[38] M. Abate, K. Gravare Silbernagel, C. Siljeholm et al., "Pathogenesis of tendinopathies: inflammation or degeneration?" Arthritis Research \& Therapy, vol. 11, no. 3, pp. 235-249, 2009.

[39] S.-C. Fu, C. Rolf, Y.-C. Cheuk, P. P. Y. Lui, and K.-M. Chan, "Deciphering the pathogenesis of tendinopathy: a three-stages process," Sports Medicine, Arthroscopy, Rehabilitation, Therapy and Technology, vol. 2, no. 1, pp. 30-41, 2010.

[40] M. A. Kahn, Ankylosing Spondylitis, Oxford University Press, New York, NY, USA, 2010.

[41] M. Benjamin, H. Toumi, D. Suzuki, K. Hayashi, and D. McGonagle, "Evidence for a distinctive pattern of bone formation in enthesophytes," Annals of the Rheumatic Diseases, vol. 68, no. 6, pp. 1003-1010, 2009.

[42] N. Maffulli, P. Sharma, and K. L. Luscombe, "Achilles tendinopathy: aetiology and management," Journal of the Royal Society of Medicine, vol. 97, no. 10, pp. 472-476, 2004.

[43] J. D. Rees, N. Maffulli, and J. Cook, "Management of tendinopathy," American Journal of Sports Medicine, vol. 37, no. 9, pp. 1855-1867, 2009.

[44] M. Benjamin and D. McGonagle, "The enthesis organ concept and its relevance to the spondyloarthropathies," Advances in Experimental Medicine and Biology, vol. 649, pp. 57-70, 2009.

[45] D. McGonagle, R. J. Wakefield, L. T. Ai et al., "Distinct topography of erosion and new bone formation in achilles tendon enthesitis: implications for understanding the link between inflammation and bone formation in spondylarthritis," Arthritis and Rheumatism, vol. 58, no. 9, pp. 2694-2699, 2008.

[46] R. J. Lories and D. L. P. Baeten, "Differences in pathophysiology between rheumatoid arthritis and ankylosing spondylitis," Clinical and Experimental Rheumatology, vol. 27, no. 4, pp. S10-S14, 2009.

[47] J. H. C. Wang and B. P. Thampatty, "Mechanobiology of adult and stem cells," International Review of Cell and Molecular Biology, vol. 271, pp. 301-346, 2008.

[48] M. L. Stoll, "Interactions of the innate and adaptive arms of the immune system in the pathogenesis of spondyloarthritis," Clinical and Experimental Rheumatology, vol. 29, no. 2, pp. 322-330, 2011.

[49] J. M. Fedorczyk, A. E. Barr, S. Rani et al., "Exposure-dependent increases in IL- $1 \beta$, substance P, CTGF, and tendinosis in flexor digitorum tendons with upper extremity repetitive strain injury," Journal of Orthopaedic Research, vol. 28, no. 3, pp. 298$307,2010$.

[50] L. M. Sconfienza, E. Silvestri, and M. A. Cimmino, "Sonoelastography in the evaluation of painful Achilles tendon in amateur athletes," Clinical and Experimental Rheumatology, vol. 28 , no. 3 , pp. $373-378,2010$.
[51] F. Batista, C. Nery, M. Pinzur et al., "Achilles tendinopathy in diabetes mellitus," Foot and Ankle International, vol. 29, no. 5, pp. 498-501, 2008.

[52] J. Zwerver, S. W. Bredeweg, and I. van den Akker-Scheek, "Prevalence of jumper's knee among nonelite athletes from different sports: a cross-sectional survey," American Journal of Sports Medicine, vol. 39, no. 9, pp. 1984-1988, 2011.

[53] J. T. Blackburn, D. R. Bell, M. F. Norcross, J. D. Hudson, and M. H. Kimsey, "Sex comparison of hamstring structural and material properties," Clinical Biomechanics, vol. 24, no. 1, pp. 65-70, 2009.

[54] R. J. Butler, H. P. Crowell, and I. M. Davis, "Lower extremity stiffness: implications for performance and injury," Clinical Biomechanics, vol. 18, no. 6, pp. 511-517, 2003.

[55] M. Rudwaleit, A. Metter, J. Listing, J. Sieper, and J. Braun, "Inflammatory back pain in ankylosing spondylitis: a reassessment of the clinical history for application as classification and diagnostic criteria," Arthritis and Rheumatism, vol. 54, no. 2, pp. 569-578, 2006.

[56] T. R. Jenkinson, P. A. Mallorie, H. C. Whitelock, L. G. Kennedy, S. L. Garrett, and A. Calin, "Defining spinal mobility in ankylosing spondylitis (AS). The Bath AS metrology index," Journal of Rheumatology, vol. 21, no. 9, pp. 1694-1698, 1994.

[57] E. Witvrouw, J. Bellemans, R. Lysens, L. Danneels, and D. Cambier, "Intrinsic risk factors for the development of patellar tendinitis in an athletic population: a two-year prospective study," American Journal of Sports Medicine, vol. 29, no. 2, pp. 190-195, 2001.

[58] J. Braun, R. van den Berg, X. Baraliakos et al., "2010 update of the ASAS/EULAR recommendations for the management of ankylosing spondylitis," Annals of the Rheumatic Diseases, vol. 70, no. 6, pp. 896-904, 2011.

[59] S. Masiero, L. Bonaldo, M. Pigatto, A. Lo Nigro, R. Ramonda, and L. Punzi, "Rehabilitation treatment in patients with ankylosing spondylitis stabilized with tumor necrosis factor inhibitor therapy. A randomized controlled trial," Journal of Rheumatology, vol. 38, no. 7, pp. 1335-1342, 2011.

[60] H. Dagfinrud, T. K. Kvien, and K. B. Hagen, "Physiotherapy interventions for ankylosing spondylitis," Cochrane Database of Systematic Reviews, no. 1, Article ID CD002822, 2008.

[61] C. Fernández-De-Las-Peñas, C. Alonso-Blanco, M. MoralesCabezas, and J. C. Miangolarra-Page, "Two exercise interventions for the management of patients with ankylosing spondylitis: a randomized controlled trial," American Journal of Physical Medicine and Rehabilitation, vol. 84, no. 6, pp. 407419, 2005.

[62] B. Vandooren, P. P. Tak, and D. Baeten, "Synovial and mucosal immunopathology in spondyloarthritis," Advances in Experimental Medicine and Biology, vol. 649, pp. 71-84, 2009.

[63] J. Peake, K. Nosaka, and K. Suzuki, "Characterization of inflammatory responses to eccentric exercise in humans," Exercise Immunology Review, vol. 11, pp. 64-85, 2005.

[64] R. J. Lories, F. P. Luyten, and K. de Vlam, "Progress in spondylarthritis. Mechanisms of new bone formation in spondyloarthritis," Arthritis Research \& Therapy, vol. 11, no. 2, p. 221, 2009.

[65] S. J. Pedersen, C. Praveena, R. G. W. Lambert, M. Østergaard, and W. P. Maksymowych, "Resolution of inflammation following treatment of ankylosing spondylitis is associated with new bone formation," Journal of Rheumatology, vol. 38, no. 7, pp. 1349-1354, 2011.

[66] J. H. C. Wang and B. P. Thampatty, "An introductory review of cell mechanobiology," Biomechanics and Modeling in Mechanobiology, vol. 5, no. 1, pp. 1-16, 2006. 
[67] H. B. Sun, Y. Li, D. T. Fung, R. J. Majeska, M. B. Schaffler, and E. L. Flatow, "Coordinate regulation of IL-1 $\beta$ and MMP-13 in rat tendons following subrupture fatigue damage," Clinical Orthopaedics and Related Research, vol. 466, no. 7, pp. 15551561, 2008.

[68] R. J. U. Lories, I. Derese, and F. P. Luyten, "Modulation of bone morphogenetic protein signaling inhibits the onset and progression of ankylosing enthesitis," The Journal of Clinical Investigation, vol. 115, no. 6, pp. 1571-1579, 2005.

[69] D. McGonagle, S. Savic, and M. F. McDermott, "The NLR network and the immunological disease continuum of adaptive and innate immune-mediated inflammation against self," Seminars in Immunopathology, vol. 29, no. 3, pp. 303-313, 2007.

[70] S. Uderhardt, D. Diarra, J. Katzenbeisser et al., "Blockade of Dickkopf (DKK)-1 induces fusion of sacroiliac joints," Annals of the Rheumatic Diseases, vol. 69, no. 3, pp. 592-597, 2010.

[71] D. Diarra, M. Stolina, K. Polzer et al., "Dickkopf-1 is a master regulator of joint remodeling," Nature Medicine, vol. 13, no. 2, pp. 156-163, 2007.

[72] D. Daoussis, S. N. C. Liossis, E. E. Solomou et al., "Evidence that Dkk-1 is dysfunctional in ankylosing spondylitis," Arthritis and Rheumatism, vol. 62, no. 1, pp. 150-158, 2010.

[73] W. P. Maksymowych, "What do biomarkers tell us about the pathogenesis of ankylosing spondylitis?" Arthritis Research and Therapy, vol. 11, no. 1, article 101, 2009.

[74] R. J. U. Lories, I. Derese, J. L. Ceuppens, and F. P. Luyten, "Bone Morphogenetic Proteins 2 and 6, expressed in arthritic synovium, are regulated by proinflammatory cytokines and differentially modulate fibroblast-like synoviocyte apoptosis," Arthritis and Rheumatism, vol. 48, no. 10, pp. 2807-2818, 2003.

[75] D. Wendling, J. P. Cedoz, E. Racadot, and G. Dumoulin, "Serum IL-17, BMP-7, and bone turnover markers in patients with ankylosing spondylitis," Joint Bone Spine, vol. 74, no. 3, pp. 304-305, 2007.

[76] M. C. Park, Y. B. Park, and S. K. Lee, "Relationship of bone morphogenetic proteins to disease activity and radiographic damage in patients with ankylosing spondylitis," Scandinavian Journal of Rheumatology, vol. 37, no. 3, pp. 200-204, 2008.

[77] J. A. Di Battista, S. Doré, J. Martel-Pelletier, and J. P. Pelletier, "Prostaglandin E2 stimulates incorporation of proline into collagenase digestible proteins in human articular chondrocytes: identification of an effector autocrine loop involving insulin-like growth factor I," Molecular and Cellular Endocrinology, vol. 123, no. 1, pp. 27-35, 1996.

[78] M. Rudwaleit, J. Listing, J. Brandt, J. Braun, and J. Sieper, "Prediction of a major clinical response (BASDAI 50) to tumour necrosis factor alpha blockers in ankylosing spondylitis," Annals of the Rheumatic Diseases, vol. 63, pp. 665-670, 2004.

[79] A. Sattler, U. Wagner, M. Rossol et al., "Cytokine-induced human IFN- $\gamma$-secreting effector-memory Th cells in chronic autoimmune inflammation," Blood, vol. 113, no. 9, pp. 19481956, 2009.

[80] N. Yeremenko, G. M. M. Rigter, I. Simon, J. D. Canete, P. P. Tak, and D. Baeten, "Identification of robust and diseasespecific stromal alterations in spondyloarthritis synovitis," Annals of the Rheumatic Diseases, vol. 70, supplement 3, aticle 67, 2011.

[81] V. Gurfinkel, T. W. Cacciatore, P. Cordo, F. Horak, J. Nutt, and R. Skoss, "Postural muscle tone in the body axis of healthy humans," Journal of Neurophysiology, vol. 96, no. 5, pp. 26782687, 2006.
[82] R. L. Lieber, Skeletal Muscle Structure, Function, and Plasticity: The Physiological Basis of Rehabilitation, Lippincott Williams \& Wilkins, Baltimore, Md, USA, 3rd edition, 2010.

[83] A. Bergmark, "Stability of the lumbar spine. A study in mechanical engineering," Acta Orthopaedica Scandinavica, Supplement, vol. 60, no. 230, pp. 5-54, 1989.

[84] L. Hansen, M. de Zee, J. Rasmussen, T. B. Andersen, C. Wong, and E. B. Simonsen, "Anatomy and biomechanics of the back muscles in the lumbar spine with reference to biomechanical modeling," Spine, vol. 31, no. 17, pp. 1888-1899, 2006.

[85] R. L. Gajdosik, "Passive extensibility of skeletal muscle: review of the literature with clinical implications," Clinical Biomechanics, vol. 16, no. 2, pp. 87-101, 2001.

[86] M. Bizzini and A. F. Mannion, "Reliability of a new, hand-held device for assessing skeletal muscle stiffness," Clinical Biomechanics, vol. 18, no. 5, pp. 459-461, 2003.

[87] R. K. Korhonen, A. Vain, E. Vanninen, R. Viir, and J. S. Jurvelin, "Can mechanical myotonometry or electromyography be used for the prediction of intramuscular pressure?" Physiological Measurement, vol. 26, no. 6, pp. 951-963, 2005.

[88] M. Tanter, J. Bercoff, A. Athanasiou et al., "Quantitative assessment of breast lesion viscoelasticity: initial clinical results using supersonic shear imaging," Ultrasound in Medicine and Biology, vol. 34, no. 9, pp. 1373-1386, 2008.

[89] A. Athanasiou, A. Tardivon, M. Tanter et al., "Breast lesions: quantitative elastography with supersonic shear imagingpreliminary results," Radiology, vol. 256, no. 1, pp. 297-303, 2010.

[90] M. Shinohara, K. Sabra, J. L. Gennisson, M. Fink, and M. L. Tanter, "Real-time visualization of muscle stiffness distribution with ultrasound shear wave imaging during muscle contraction," Muscle and Nerve, vol. 42, no. 3, pp. 438-441, 2010. 


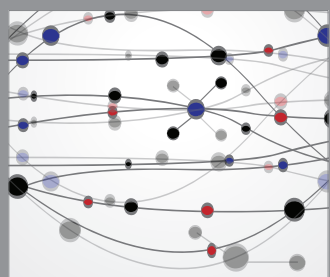

The Scientific World Journal
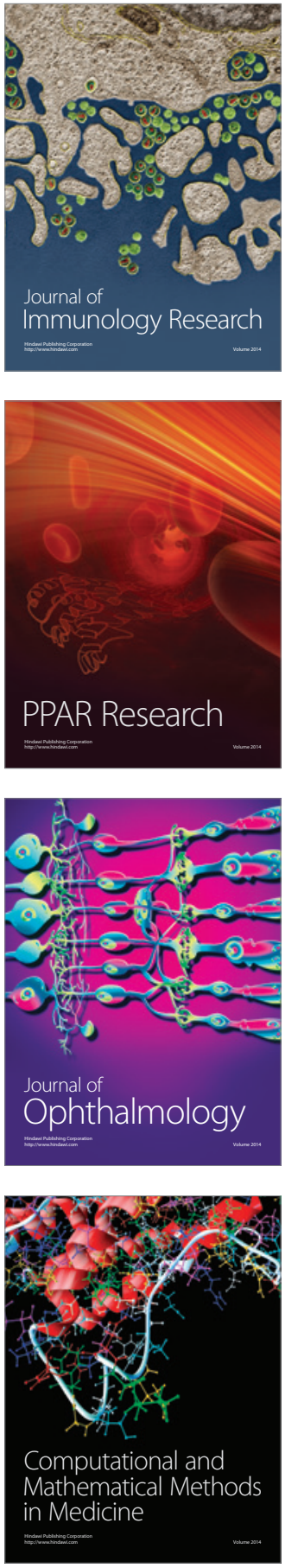

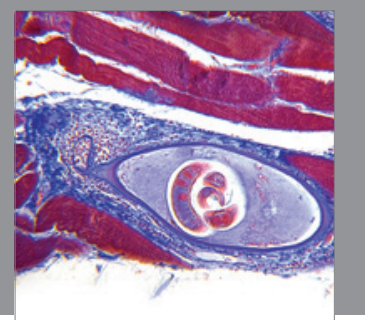

Gastroenterology

Research and Practice
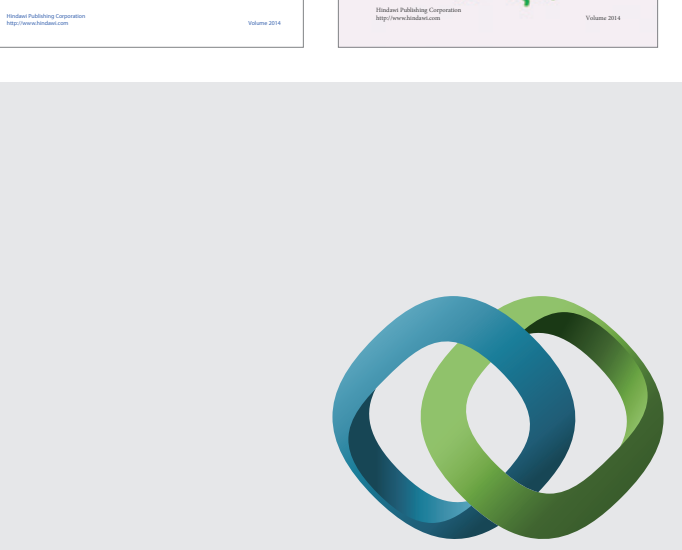

\section{Hindawi}

Submit your manuscripts at

http://www.hindawi.com
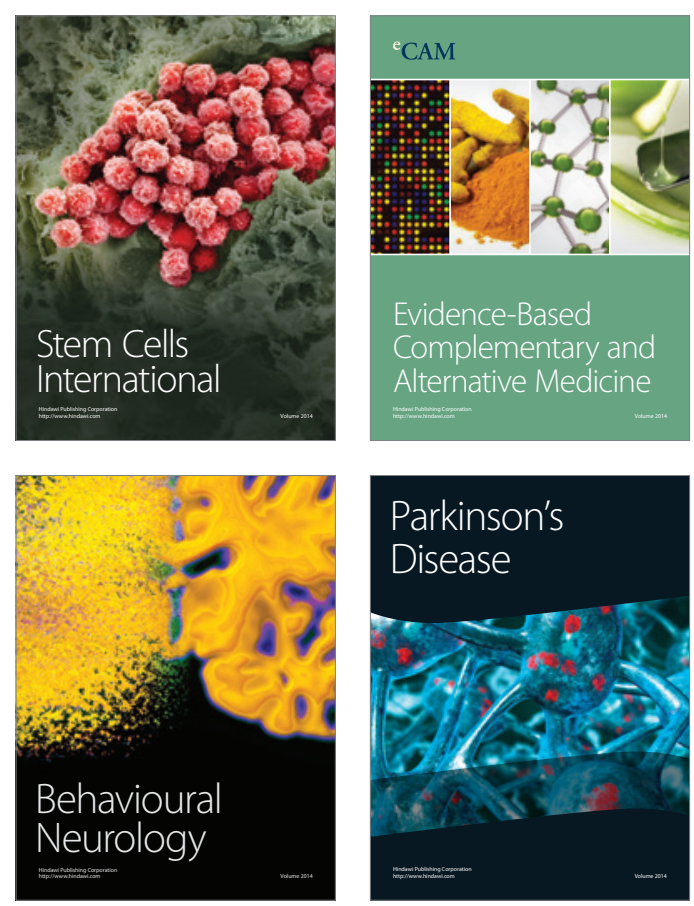

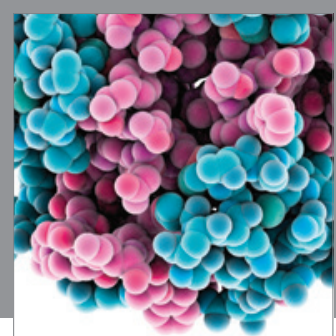

Journal of
Diabetes Research

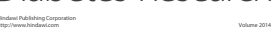

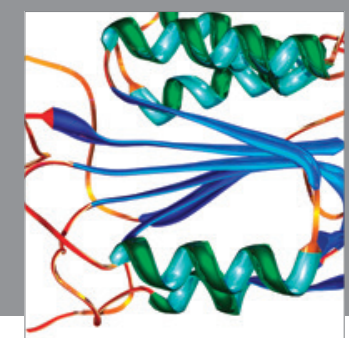

Disease Markers
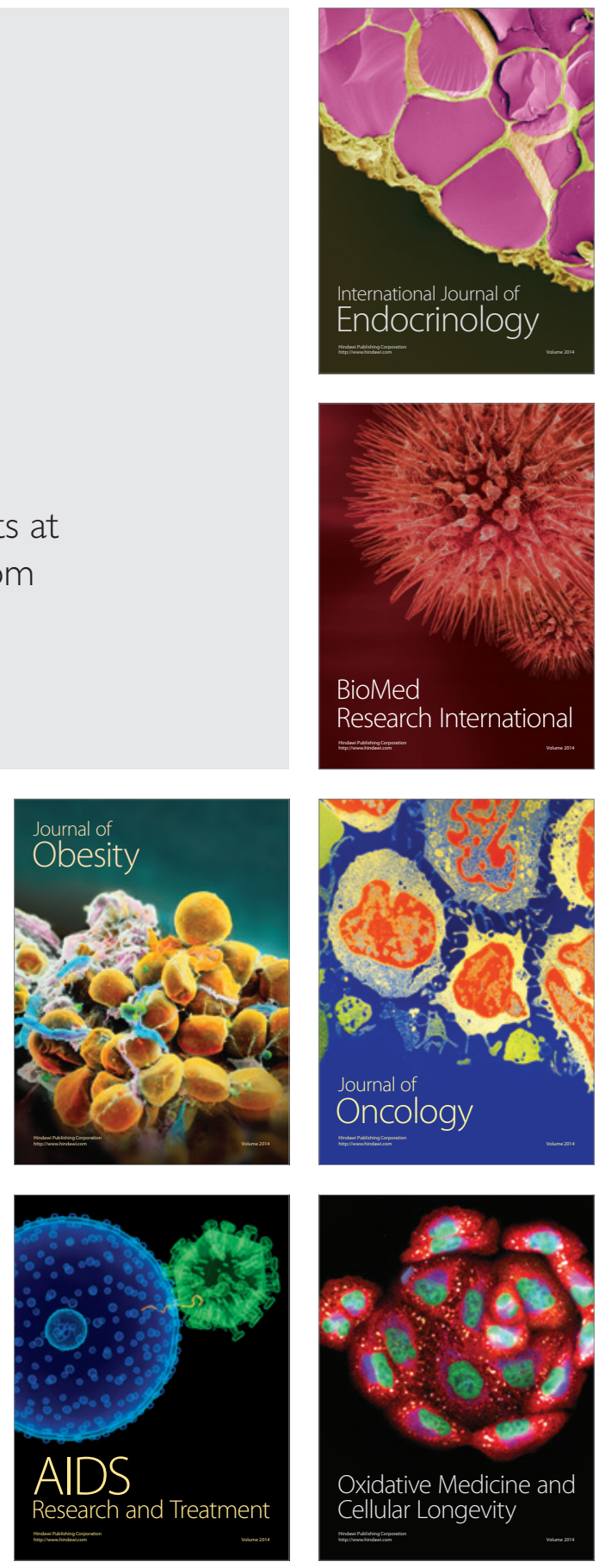